\title{
Functional Anatomy of Arithmetic and Word Reading and its Relationship to Age
}

Tanya M. Evans, ${ }^{a}$ D. Lynn Flowers, ${ }^{\text {ab }}{ }^{\text {Megan M. Luetje, }}{ }^{a}$ Eileen Napoliello ${ }^{\mathrm{a}}$ and Guinevere F. Eden $^{\mathrm{a}}$

a Center for the Study of Learning, Department of Pediatrics, Georgetown University Medical Center, Suite150 Building D, 4000 Reservoir Road NW, Washington, DC 20057 USA

b Wake Forest University Baptist Medical Center, Medical Center Boulevard, Winston-Salem, NC

Correspondence to:

Guinevere Eden, D.Phil.,

Center for the Study of Learning,

Georgetown University Medical Center,

BOX 571406,

Suite 150, Building D,

4000 Reservoir Road, NW,

Washington, DC 20057, USA

E-mail: edeng@georgetown.edu

(C) 2016. This manuscript version is made available under the Elsevier user license 


\begin{abstract}
Arithmetic and written language are uniquely human skills acquired during early schooling and used daily. While prior studies have independently characterized the neural bases for arithmetic and reading, here we examine both skills in a single study to capture their shared and unique cognitive mechanisms, as well as the role of age/experience in modulating their neural representations. We used functional MRI in 7- to 29-year-olds who performed single-digit subtraction, single-digit addition, and single-word reading. Using a factorial design, we examined the main effects of Task (subtraction, addition, reading) and Age (as a continuous variable), and their interactions. A main effect of Task revealed preferential activation for subtraction in bilateral intraparietal sulci and supramarginal gyri, right insula, inferior frontal gyrus, and cingulate. The right middle temporal gyrus and left superior temporal gyrus were preferentially active for both addition and reading, and left fusiform gyrus was preferentially active for reading. A main effect of Age revealed increased activity in older participants in right angular gyrus, superior temporal sulcus, and putamen, and less activity in left supplementary motor area, suggesting a left frontal to right temporo-parietal shift of activity with increasing age/experience across all tasks. Interactions for Task by Age were found in right hippocampus and left middle frontal gyrus, with older age invoking greater activity for addition and at the same time less activity for subtraction and reading. Together, in a study conducted in the same participants using similar task and acquisition parameters, the results reveal the neural substrates of these educationally relevant cognitive skills in typical participants in the context of age/experience.
\end{abstract}

Keywords: arithmetic, reading, development, addition, subtraction, language 


\section{INTRODUCTION}

Mastery of reading and arithmetic is crucial for academic achievement. As such, there is an urgent need to understand how both skills become successfully established in the brains of children and adults who master them well. Reading and arithmetic are distinctive in our repertoire of cognitive skills because they are relatively recent cultural inventions that are uniquely human and are explicitly taught over a protracted period of time via formal schooling. It has been proposed that reading and arithmetic rely on brain mechanisms that have an evolutionary history (e.g., language), combined with those that become redirected de novo through learning to accommodate these tasks in a framework described as "neuronal recycling" (Dehaene and Cohen, 2007).

Reading relies on the left temporo-parietal and inferior frontal cortices for aspects that it shares with spoken language, such as phonological processing (Price, 2012), while the left occipito-temporal cortex in the ventral visual stream, which subserves object processing, demonstrates specialization for visual word form recognition in skilled readers (Cohen and Dehaene, 2004; Dehaene and Cohen, 2011; Glezer et al., 2009; McCandliss et al., 2003). Arithmetic is supported by a bilateral fronto-parietal network (Arsalidou and Taylor, 2011; Kaufmann et al., 2011; Menon, 2010; Nieder and Dehaene, 2009), which is modulated based on task demands. As posited by the "triple-code model" of number processing (Dehaene, 1992; Dehaene and Cohen, 1995; Dehaene et al., 2003), particular brain regions are assigned to specific systems of representations of numerical information (quantitative, verbal, and visual). Of these, the quantitative system is unique to numerical processing, whereas the verbal and visual systems share aspects with language processing. Within the domain of arithmetic, the extent to which each operation calls on these systems varies. For example, arithmetic problems with greater 
quantitative demands (e.g., subtraction) demonstrate increased reliance on bilateral frontoparietal regions (Rosenberg-Lee et al., 2011a; De Smedt et al., 2011), while arithmetic problems that are solved via more efficient verbal retrieval strategies (such as small-digit addition and multiplication) engage left angular gyrus (AG), and middle temporal and inferior frontal cortex (Dehaene et al., 2003; Grabner et al., 2009; Lee, 2000; Prado et al., 2011). As such, the verbal aspect of arithmetic retrieval shares some neural mechanisms with language.

Associations between some aspects of language and arithmetic have long been supported by behavioral, brain lesion, and more recently, functional brain imaging studies. In behavioral research, a dual-task experiment showed a double dissociation for the interference of a verbal task (phonological suppression) and a visuospatial task (visuospatial suppression) with two types of arithmetic problems (multiplication and subtraction), respectively, suggesting that these two operations are mediated by verbal codes rather than visuospatial codes (Lee and Kang, 2002). Further, verbally mediated retrieval of arithmetic facts is correlated with phonological awareness (Barnes et al., 2014; De Smedt et al., 2010; Hecht et al., 2001; Simmons and Singleton, 2008), a skill that is integral to reading (Melby-Lervåg et al., 2012). Double dissociations reported in patients with brain lesions leading to acalculia (Anderson et al., 1990; Lee, 2000) support the notion of differential involvement of brain systems that are task dependent. For example, lesions to left perisylvian cortex (associated with language processing) are associated with degraded multiplication but spared subtraction. On the other hand, intraparietal cortical lesions interfere with subtraction but not multiplication (Cohen et al., 2000; Dehaene and Cohen, 1997; van Harskamp and Cipolotti, 2001; van Harskamp et al., 2002; Mihulowicz et al., 2014). Brain imaging studies have confirmed these functional specializations for arithmetic tasks and the unique relationships that exist between some specific aspects of arithmetic and left hemisphere 
language areas. For example, left middle temporal and inferior frontal gyrus (IFG) regions that respond during phonological processing (using a phonological localizer task) demonstrate greater activity during multiplication relative to subtraction (Prado et al., 2011). Other research has shown a positive relationship between mathematical competence and activity in the left AG during multiplication tasks (Grabner et al., 2007). These same investigators also report stronger activation of the left AG during arithmetic problem solving for which participants reported using retrieval rather than quantitative strategies (Grabner et al., 2009).

Neuroanatomical investigations of white matter tracts also support the idea that there is specialization for specific arithmetic operations in various brain regions and that amongst these, some, but not all, have a relationship with regions involved in language processing. In a study of unilateral stroke patients, lesion density in the left arcuate fasciculus was shown to correspond with addition and multiplication impairments, but patients had preserved subtraction skills (Mihulowicz et al., 2014). In typically reading children, a relationship has been demonstrated between white matter integrity of left fronto-parietal aspects of the arcuate fasciculus and arithmetic competence in addition and multiplication, but not subtraction and division (Van Beek et al., 2014). Interestingly, this tract is known to be related to reading ability (Odegard et al., 2009; Rauschecker et al., 2009; Thiebaut de Schotten et al., 2012; Yeatman et al., 2011) and is compromised in the reading disability dyslexia (Vandermosten et al., 2012). As such, it is notable that the relationship between arithmetic supported by verbal retrieval and white matter integrity shown by Van Beek and colleagues disappears when non-word reading skills are controlled for, again suggesting that phonological processing skills (used for decoding of nonwords) play a mediating role in addition and multiplication abilities. Together, these behavioral, clinical, and neuroimaging studies support the notion of task-specific brain regions underlying 
different arithmetic tasks and reading, yet at the same time speak to some shared brain circuitry for specific language tasks and specific arithmetic operations. A more detailed characterization is important for advancing our understanding of brain-based models of learning disabilities in these domains (i.e. dyslexia and dyscalculia) as well as the broader versus more specific impact of instruction.

Naturally, inquiries into the neural bases of reading and arithmetic are highly dependent on the age of the participants, which in turn is associated with expertise of these skills. The brain undergoes profound changes during the course of development. With age, the systems supporting executive function, language and other cognitive tasks mature, and these domain general constructs allow for the mastery of more complex cognitive skills (Shaw et al., 2006). For example, gray matter has been shown to decrease with increasing age in prefrontal and parietal cortices (Sowell et al., 2003). At the same time, experience-dependent learning has an effect on this developmental trajectory, as illustrated by the relationship between increased phonological awareness skills and increases in gray matter in the left IFG (Lu et al., 2007). Reading and arithmetic are learned skills, and their unique and shared neural circuits are therefore likely to change over time. Further, brain areas that constitute, for example, the reading network are brought together as a function of learning to read and not due to any other pre-existing privileged relationship (Vogel et al., 2013). In developmental studies, independent lines of investigation have examined age-related changes during reading (Schlaggar et al., 2002; Turkeltaub et al., 2003; for review see Church et al., 2008; Pugh et al., 2001; Schlaggar and McCandliss, 2007) or arithmetic (Kawashima et al., 2004; Kucian et al., 2008; Qin et al., 2014; Rivera et al., 2005). While no studies to date have looked into the developmental patterns of both in the same subjects, parallel meta-analyses of reading and numerical processing in children show that 
children are likely to engage a left-lateralized frontal, temporo-parietal, and occipito-temporal network during reading while also engaging frontal cortex during numerical comparison, a range of arithmetic tasks, and algebraic problem solving (Houdé et al., 2010). The authors suggest from these analyses in children that, while similar patterns are seen in adults during reading, children appear to rely more heavily than adults on frontal regions during numerical processing (studies of specific arithmetic tasks were not included in the meta-analysis). Another meta-analysis that focused on numerical processing studies in children identified brain regions that include bilateral fronto-parietal cortices during arithmetic problem solving (Kaufmann et al., 2011), but again, did not compare these regions to adult brains. Empirical studies comparing children with adults are mixed, with some showing increased reliance on left posterior parietal cortex with age (Rivera et al., 2005), while others have demonstrated greater engagement of bilateral posterior parietal and frontal cortices in adults relative to children for arithmetic (Kawashima et al., 2004; Kucian et al., 2008; Qin et al., 2014). For reading, some developmental work has indicated a posterior-toanterior shift with age within the left hemisphere (Turkeltaub et al., 2003). It has also been suggested that there is increased specialization of occipito-temporal cortex over development (Pugh et al., 2001), which is consistent with less activity in adults relative to children in left angular and supramarginal gyri (Church et al., 2008). A recent meta-analysis also revealed greater reliance on left superior temporal cortex by children and greater reliance on posterior occipito-temporal/cerebellar regions by adults (Martin et al., 2015). However, longitudinal studies have yet to be conducted to directly test for these developmental patterns. As such, there are some inconsistencies in the few studies aimed at identifying the developmental trajectories of arithmetic or reading. 
Nevertheless, the results from these disparate studies suggest two things: First, while reading and arithmetic largely engage different brain regions, they do overlap in the left middle temporal, inferior parietal, and inferior frontal cortices, especially when the arithmetic tasks involve verbally mediated retrieval strategies. Second, neuroplastic changes accompany the acquisition of expertise in each of these skills. These are constrained by the underlying anatomical developmental stages (Giedd et al., 1999) and modulated by the task-specific demands. Here, we directly address the modulation that occurs in brain activity due to task- and age-specific aspects of math processing and reading. We examined brain activity in a sample of typically developing children, adolescents, and adults carefully evaluated for their typical reading and arithmetic abilities.

We employed similarly designed tasks to examine arithmetic and single-word processing, suitable for both children and adults. Naturally, reading and arithmetic differ in the way in which they are executed and in this study we tried to strike a balance to respect these differences whilst at the same time reaching similarity in task design and execution. In a classroom or educational testing setting, arithmetic is typically conducted during silent pencil and paper tasks, while reading can be performed silently or aloud, on single words and on connected text. The constraints of MRI and other aspects of experimental design have led to covert single word processing becoming the favored task, as is reflected in the corpus of functional MRI studies of reading as exemplified in a recent meta-analysis of reading in children and adults (Martin et al., 2015a). As such our goal was to employ a task that elicits activity consistent with this published literature, including areas thought to be involved in visual word form recognition as well as phonological processing. The implicit reading task employed by us (Krafnick et al., 2016; Olulade et al., 2013a, 2015, Turkeltaub et al., 2003, 2004) and others (Paulesu et al., 2001; Price 
et al., 1996) meets this need. For example, group maps of children performing this task (Olulade et al., 2013a) is highly consistent with those published for a meta-analysis of reading based on 20 pediatric studies (Martin et al., 2015a).

Single-digit arithmetic was separated into the operations of addition (more likely to invoke verbal retrieval subserved by areas involved in language processing) and subtraction (more likely to involve quantitative procedures subserved by intraparietal cortex). We first performed analyses for each of these three tasks in a group of subjects ranging in age from 7 to 29 years, for ease of comparison with prior studies. To test our predictions, we then combined the data from all three tasks and examined the effect of Task (subtraction, addition, and reading) via an analysis of covariance (ANCOVA), which also served to reveal the effect of Age (as a continuous variable). Depending on task and processing demands, neural activity in relevant brain structures can either increase (Lewandowska et al., 2010; Vogan et al., 2016) or decrease (Church et al., 2008; Dunst et al., 2014) as expertise develops with age. Here our predictions align with increased activity associated with increased engagement and experience, though we acknowledge that decreases in activity could be attributed to increased neural efficiency or change in strategy (e.g. as in reading where development might be associated with disengagement of superior temporal lobe structures for phonological processing and greater engagement of occipito-temporal cortex for more automatic word form recognition). We predicted that main effects for Task would involve greater activity in bilateral frontal and parietal cortices during subtraction, reflecting reliance on quantitative circuitry. We also predicted coactivation during addition and word processing in left inferior parietal, middle/superior temporal, and inferior frontal cortices, due to mutual involvement of verbal systems, consistent with the behavioral, patient, and neuroimaging work described above. Further, we expected the 
engagement of these to be generally more pronounced with increasing age due to maturation and greater expertise with these skills. The Task by Age interaction would reveal those areas where any of these tasks are uniquely modulated by age. We predicted increased activity with increasing age in left inferior frontal cortex during reading and addition, and increased activity with increasing age in parietal cortices during subtraction.

\section{METHODS}

\subsection{Subjects}

Thirty subjects participated in this study. The participants ranged in age from 7 to 29 years old. The Georgetown University Institutional Review Board approved all experimental procedures, and written informed consent was obtained from each individual and from the legal guardian for subjects less than 18 years of age. All participants were monolingual English speakers and in good physical health and constituted 12 females and 18 males.

\subsection{Neuropsychological Battery}

All subjects were evaluated using standardized measures and performed in or above the normal range (> 85 standard score) on all of these. We measured IQ (Wechsler Abbreviated Scale of Intelligence: WASI-FSIQ; Wechsler, 1999), and the group had a mean (SD) of 119.5 (11.3) on the Performance IQ and 115.3 (9.6) on the Verbal IQ portions. In the domain of arithmetic, we measured untimed computation (Woodcock-Johnson III Tests of Achievement: W-J Calculation; Woodcock et al., 2001; mean: 118.0 (8.8)), arithmetic speed (W-J Math Fluency; mean: 108.6 (12.1)), and problem solving (W-J Applied Problems; mean: 117.0 (9.3)). For reading we assessed single real word reading (W-J Word Identification; mean: 114.5 (9.1)). There were no significant Pearson correlations between subject age and standardized scores of 
all of these measures ( $p>0.05$ ), allowing us to be confident that any age-dependent differences in brain activity were not driven by differences in skill level between younger and older participants.

\subsection{Arithmetic Task}

Subjects performed single-digit calculation verification tasks in the scanner for addition or subtraction in separate blocks. Each task consisted of a two-operand equation with a singledigit resultant (e.g., $2+3=5$ or $7-4=3$ ), and subjects indicated via right or left thumb button press whether the resultant was correct or incorrect. For incorrect experimental stimuli, resultants were either one more or one less than the correct answer (e.g., $2+3=6$ ). In the active control conditions, one of the operands and the resultant were replaced by pseudofont characters, and subjects indicated whether these characters on either side of the equal sign were the same (e.g., $ト-5=ト$ ) or different (e.g., $4+\boldsymbol{A}=ट$ ). Each task (subtraction and addition) had their unique control stimuli. There were 10 unique stimuli for each condition (addition, addition control, subtraction, and subtraction control). We chose to present the entire equation, with solution, to mirror the presentation of the reading task. Addition and subtraction were acquired in alternating blocks within the same experimental run. Correct and incorrect arithmetic experimental problems (50\% correct, $50 \%$ incorrect) and same and different control problems (50\% correct, $50 \%$ incorrect) were randomized within each block. This task has been used in previous studies of children (Evans et al., 2014). Further task details are provided below in section 2.5.

\subsection{Reading Task}

We employed a word processing task that has a similar presentation format to the arithmetic tasks described above. In this task, subjects viewed single five-letter, low frequency 
(KF 8.1, SD 6.1) words and indicate by button press the presence of a tall letter (e.g., there is a tall letter, 't', in the word 'stoop'; there is not a tall letter in the word 'marry'). In a control task, words were replaced by strings of pseudofont characters, and subjects indicated whether they saw a tall character or not. The comparison of these two conditions reveals brain areas engaged in orthographic, phonological, and semantic aspects of single-word reading (Price et al., 1996). Pseudofont strings matched word stimuli for length, size, and location of tall letters/characters. To avoid confusion, words containing the letters $\mathrm{i}$ and $\mathrm{j}$ were excluded. The presence of a tall letter/character in a word/pseudofont string (50\% tall, 50\% no tall letter) was randomized within each block. This implicit reading task was originally developed by Price and colleagues (Price et al., 1996), and we have used it in children in previous studies (Olulade et al., 2013b, 2015, Turkeltaub et al., 2003, 2004). Further task details follow below in Section 2.5.

A post-test was administered following the fMRI scan, outside of the scanner, to gauge if the children had been processing the stimuli implicitly. The test consisted of a list of forty stimuli, half of which had been presented during the scan and the other half of which had not. Subjects indicated using a checkmark whether they believed they had seen the stimulus in the scanner or not (Turkeltaub et al., 2003).

\section{5 fMRI Acquisition}

A block-design paradigm was utilized with two 4-minute, 27-second runs for each task consisting of 4 blocks with 10 trials per block for each condition. The stimulus duration was 1.2 seconds, and the inter-stimulus interval was 3 seconds. Stimuli were presented in black font on a white screen, and a black fixation cross was presented during the inter-stimulus interval. Blocks were interspersed with five 18-second blocks of rest (during which the black fixation cross was presented), with an additional 6 seconds coming at the beginning and 3 seconds at the end of 
each run. This resulted in the collection of a total of 28 whole-head EPI volumes for each arithmetic condition (subtraction, addition) and 56 for reading. To equate for these different numbers of volumes for data analysis, analysis was performed on data acquired during two runs for the arithmetic tasks and one run for the reading task. The selection of the single reading run was based on a randomization procedure, which ensured that $50 \%$ of the runs represented the first run and $50 \%$ the second run. Scanning was done on a 3.0 Tesla Siemens Tim Trio Magnetom scanner with the following acquisition parameters: $\mathrm{TR}=3000 \mathrm{~ms}$, TE $=30 \mathrm{~ms}, 64 \mathrm{x}$ 64 matrix, 192 mm FOV, 50 axial slices, 3.0 x 3.0 x 2.8mm voxels. Structural 3-D T1 images were acquired (FOV read: 256; phase: 256; slices: 160; slice resolution: $1 \mathrm{~mm}$ ) for each participant yielding images with a voxel size of $1 \times 1 \times 1 \mathrm{~mm}$, and used to coregister the functional data.

\section{6 fMRI Analysis}

\subsubsection{First Level}

To account for any T1 saturation effects, the first five scans of each run were removed prior to analysis. Using Statistical Parametric Mapping (SPM8, Wellcome Department of Cognitive Neurology, London) for all analyses, we first preprocessed the data: motion correction by realigning to the mean functional image, normalization to the standard Montreal Neurological Institute (MNI) EPI template, and smoothing using an $8 \mathrm{~mm}^{3}$ Gaussian kernel. While there have been some concerns for normalization of pediatric data to adult templates (Wilke et al., 2002, 2003), this has primarily been for T1-weighted images used for tissue classification. Our current study's use of normalization to the MNI EPI template for fMRI data was consistent with our prior pediatric studies (Krafnick et al., 2016; Olulade et al., 2013b, 2013c, 2015) based on the 
feasibility of transforming pediatric brains into adult stereotactic space (Burgund et al., 2002; Kang et al., 2003).

We then modeled the hemodynamic response function using the general linear model during performance of the arithmetic operations of (a) subtraction and (b) addition, as well as (c) single-word reading, regressing out global mean signal and 6 motion parameters (roll, pitch, yaw, $x, y, z)$ as covariates of no interest. Only subjects with less than $3 \mathrm{~mm}$ maximum displacement across all functional runs were included in the analysis, and all subjects met this criterion. Contrast maps were generated for each subject for the first (within-group) analyses using (1) Subtraction > Subtraction Active Control Condition, (2) Addition > Addition Active Control Condition, and (3) Reading > Reading Active Control Condition, and then for the second (ANCOVA) analyses using (4) Subtraction > Fixation, (5) Addition > Fixation and (6) Reading $>$ Fixation.

\subsubsection{Group Maps For Each of the Three Tasks}

To examine activity elicited by each task, we generated group maps using one-sample ttests. For ease of comparison with prior studies, maps were generated for subtraction, addition, and reading versus the respective active control task. The reaction time difference score for each task relative to its respective control task was entered for each subject as a covariate of no interest. A height threshold of $p<0.005$ with a FDR cluster-level extent threshold of $p<0.05$ was implemented in SPM. All coordinates are reported using MNI convention. Using the SPM Anatomy Toolbox (Eickhoff et al., 2005; 2007), the locations of the peaks of the activations were also assigned to the most likely cytoarchitectonic areas using the maximum probability map (MPM). Cytoarchitectonic maps and anatomical labels for parietal cortex come from Caspers et al. (2006) and Choi et al. (2006), which delineate three subdivisions for intraparietal sulcus (IPS; 
hIP1, hIP2, hIP3), five for supramarginal gyrus (SMG; PF, PFcm, PFm, PFop, and PFt), and two for $\mathrm{AG}$ (PGa and PGp).

\subsubsection{ANCOVA}

A whole-brain random-effects full factorial Task x Age analysis of covariance (ANCOVA) was conducted on the fMRI data with Task (subtraction, addition, and reading) as a within-subjects factor and Age as a continuous variable. In this analysis, the experimental tasks were contrasted with a common baseline consisting of passive fixation to address potential concerns that the active control task (Task B") is not the same in both children and adults (Church et al., 2010). The average reaction time for each task was entered for each subject as a covariate of no interest. F-maps were generated to determine presence of main effects and interactions. Thresholds of $\mathrm{p}<0.005$ (height) and $\mathrm{p}<0.05$ (extent) were implemented with familywise error correction using a nonstationary suprathreshold cluster-size approach based on Monte Carlo simulations (Nichols and Hayasaka, 2003). Average percent signal change was extracted from each significant cluster for each relevant condition using MarsBar (Brett et al., 2002) to examine the direction of the effects.

\section{RESULTS}

\subsection{In-Scanner Behavioral Performance}

Table 1 provides accuracy and reaction time data for the three tasks and their respective active control tasks from the scans that were used to generate the fMRI group maps for subtraction, addition, and reading. Next, the in-scanner behavioral data were analyzed in ways mirroring the fMRI factorial analyses, that is, subjected to an ANCOVA with Task (subtraction, addition, reading) as a within-subjects factor and Age as a continuous variable. For accuracy, 
there was no significant main effect of Task $\left(F(1,86)=1.65, p=0.20, \eta^{2}=0.04\right)$. There was a significant main effect of Age $\left(F(1,86)=39.65, p<0.001, \eta^{2}=0.30\right)$, with accuracy increasing with age. There was no Age $\mathrm{x}$ Task interaction $\left(\mathrm{F}(1,86)=2.39, \mathrm{p}=0.10, \eta^{2}=0.04\right)$ for accuracy. For reaction time, there was a significant main effect of Task $(F(1,86)=21.04, p<$ $\left.0.001, \eta^{2}=0.33\right)$, with reaction times being faster for reading compared with both addition ( $\mathrm{p}<$ 0.001) and subtraction $(\mathrm{p}<0.001)$, and reaction times also being faster for addition than subtraction $(\mathrm{p}<0.01)$. There was also a main effect of Age $\left(F(1,86)=55.58, \mathrm{p}<0.001, \eta^{2}=\right.$ 0.26), with faster responses with greater age. Finally, there was an Age x Task interaction (F $\left.(1,86)=34.24, p<0.001, \eta^{2}=0.33\right)$, with faster response times with greater age in both addition and subtraction.

\subsection{Post-Scanner Recognition Test}

Our post-scanner forced-choice pencil and paper recognition test, confirmed as in previous work (Turketaub et al., 2003), that there was implicit processing of the word stimuli. Subjects were able to accurately discriminate word stimuli presenting during scanning from matched distractors (i.e. significantly higher accuracy for words, $63 \%$, relative to false font stimuli, 45\%; $\mathrm{p}<0.0001)$. 
Table 1. In-Scanner Behavioral Performance on Arithmetic and Reading Tasks

\begin{tabular}{lcrr}
\hline \multirow{3}{*}{ Subtraction } & & Mean & SD \\
\cline { 2 - 3 } Control & Accuracy & $93.08 \%$ & 11.08 \\
& Reaction Time & $1708.08 \mathrm{~ms}$ & 796.43 \\
& Accuracy & $96.83 \%$ & 4.25 \\
\multirow{4}{*}{ Addition } & Reaction Time & $1102.82 \mathrm{~ms}$ & 247.03 \\
& & & \\
Control & Accuracy & $96.92 \%$ & 4.53 \\
& Reaction Time & $1312.40 \mathrm{~ms}$ & 486.68 \\
& Accuracy & $98.00 \%$ & 4.66 \\
\multirow{3}{*}{ Reading } & Reaction Time & $1055.40 \mathrm{~ms}$ & 205.08 \\
\multirow{3}{*}{ Control } & & & \\
& Accuracy & $93.59 \%$ & 9.63 \\
& Reaction Time & $798.58 \mathrm{~ms}$ & 135.51 \\
& Accuracy & $96.01 \%$ & 7.14 \\
& Reaction Time & $788.37 \mathrm{~ms}$ & 160.90 \\
\hline
\end{tabular}

\section{2 fMRI Results: Group Maps of Subtraction, Addition, and Reading in all Subjects}

\subsubsection{Subtraction > Active Control Condition}

As anticipated, a within-group analysis for all subjects for subtraction (subtraction > subtraction control) revealed activation in bilateral SMG/AG (PFm/hIP1/BA 40/7) extending into IPS. There was also activation in right IFG (BA 45), left medial frontal gyri/supplementary motor area (SMA; BA 6/32; extending into the right hemisphere), and left cerebellum (lobule VI). This bilateral fronto-parietal network is consistent with the quantitative strategies necessary to solve subtraction problems. See Table 2 and Figure 1A.

\subsubsection{Addition > Active Control Condition}

This within-group analysis for all subjects for the operation of addition (addition > addition control) revealed significant clusters in the left SMA (BA 6/32) and bilateral middle frontal gyri (MFG; BA 44/46; extending into IFG in the left and superior frontal gyrus in the right hemisphere) in all subjects. This is consistent with what has been shown previously for addition problem solving. See Table 2 and Figure 1B. 


\subsubsection{Reading > Active Control Condition}

In all subjects, the implicit reading task relative to its control condition activated left superior/middle temporal gyri (STG/MTG; BA 21/22) and IFG (BA 44) within the typical leftlateralized network associated with reading. See Table 2 and Figure 1C.

Figure 1. Within-Group Maps of Subtraction, Addition, and Reading in All Subjects

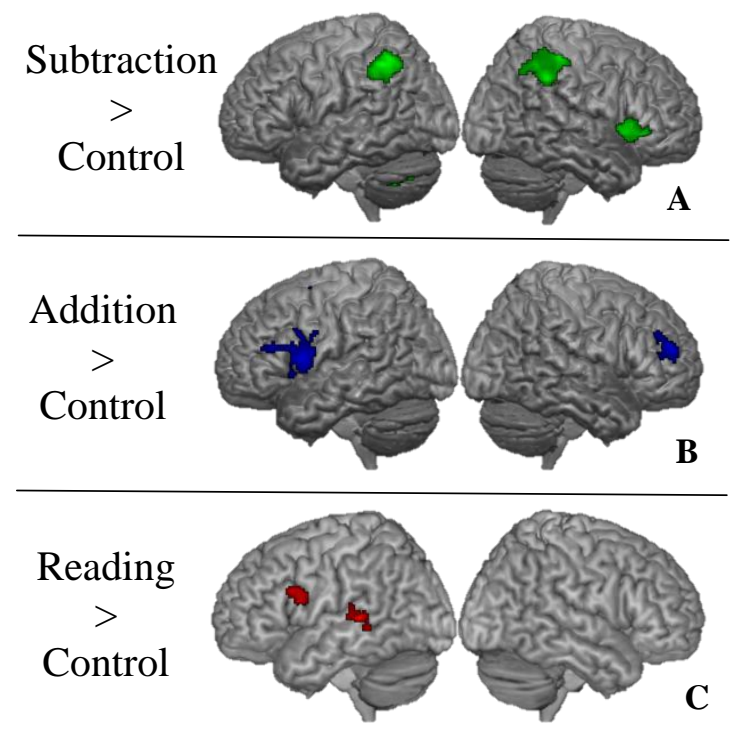

Figure 1 displays statistical within-group whole-brain maps rendered onto a structural MRI scan for all subjects for A. SUBTRACTION (subtraction task> subtraction active control task) in green, B. ADDITION (addition task > addition active control task) in blue, and C. READING (reading task > reading active control task) in red; $p<0.005$ height threshold, $p<0.05$ clusterlevel corrected extent threshold. Details on the anatomical location and significance of these results can be found in Table 2. 
Table 2. Within-Group Maps for All Subjects for Arithmetic and Reading Tasks

\begin{tabular}{|c|c|c|c|c|c|}
\hline \multirow[t]{2}{*}{ Anatomical Region } & \multicolumn{3}{|c|}{$\begin{array}{l}\text { Peak MNI } \\
\text { Coordinates }\end{array}$} & \multirow[t]{2}{*}{ Z Score } & \multirow{2}{*}{$\begin{array}{l}\text { Volume } \\
\text { (voxels) }\end{array}$} \\
\hline & $\mathbf{x}$ & $\mathbf{y}$ & $\mathbf{z}$ & & \\
\hline \multicolumn{6}{|l|}{ Subtraction > Control Task } \\
\hline Right supramarginal/angular gyri & 50 & -42 & 38 & 4.46 & 795 \\
\hline Right inferior frontal gyrus & 40 & 14 & 0 & 4.39 & 588 \\
\hline Left cerebellum & -30 & -58 & -40 & 4.11 & 407 \\
\hline $\begin{array}{l}\text { Left supramarginal/angular gyri/intraparietal } \\
\text { sulcus/superior parietal lobe }\end{array}$ & -50 & -48 & 46 & 4.74 & 635 \\
\hline $\begin{array}{l}\text { Left (extending into right) medial frontal } \\
\text { gyrus/supplementary motor area }\end{array}$ & -4 & 12 & 50 & 4.30 & 1064 \\
\hline \multicolumn{6}{|l|}{ Addition > Control Task } \\
\hline Right middle/superior frontal gyri & 30 & 50 & 20 & 4.23 & 320 \\
\hline Left inferior/middle frontal gyri & -30 & 2 & 8 & 4.46 & 846 \\
\hline Left supplementary motor area & -8 & 14 & 56 & 4.98 & 581 \\
\hline \multicolumn{6}{|l|}{ Reading > Control Task } \\
\hline Left superior/middle temporal gyri & -58 & -34 & 6 & 4.77 & 103 \\
\hline Left inferior frontal gyrus & -46 & 8 & 18 & 4.27 & 148 \\
\hline
\end{tabular}

\section{4 fMRI Results: Factorial Analysis}

\subsubsection{Main Effect of Task}

The Task x Age ANCOVA conducted on the fMRI data with Task (subtraction, addition, reading) as a within-subjects factor and Age as a continuous variable revealed a main effect of Task in ten clusters. The following clusters were preferentially activated for subtraction: right SMG extending into the IPS (hIP3/PFt), insula, IFG (BA 44/45), and cingulate, and left SMG (PF) and IPS (hIP1), fitting with quantitative procedure-based arithmetic. Clusters in the right MTG and left STG showed greater activity during addition and reading relative to subtraction. These results for addition lend further support to the role of the temporal cortex in verbally mediated tasks, including retrieval-based arithmetic. The reading task preferentially (relative to subtraction and addition) engaged clusters in bilateral precuneus and in left fusiform gyrus 
extending into parahippocampal gyrus (BA 36/37), consistent with the use of ventral visual cortex for word processing. See Table 3 and Figure 2. 
Figure 2. Main Effect of Task

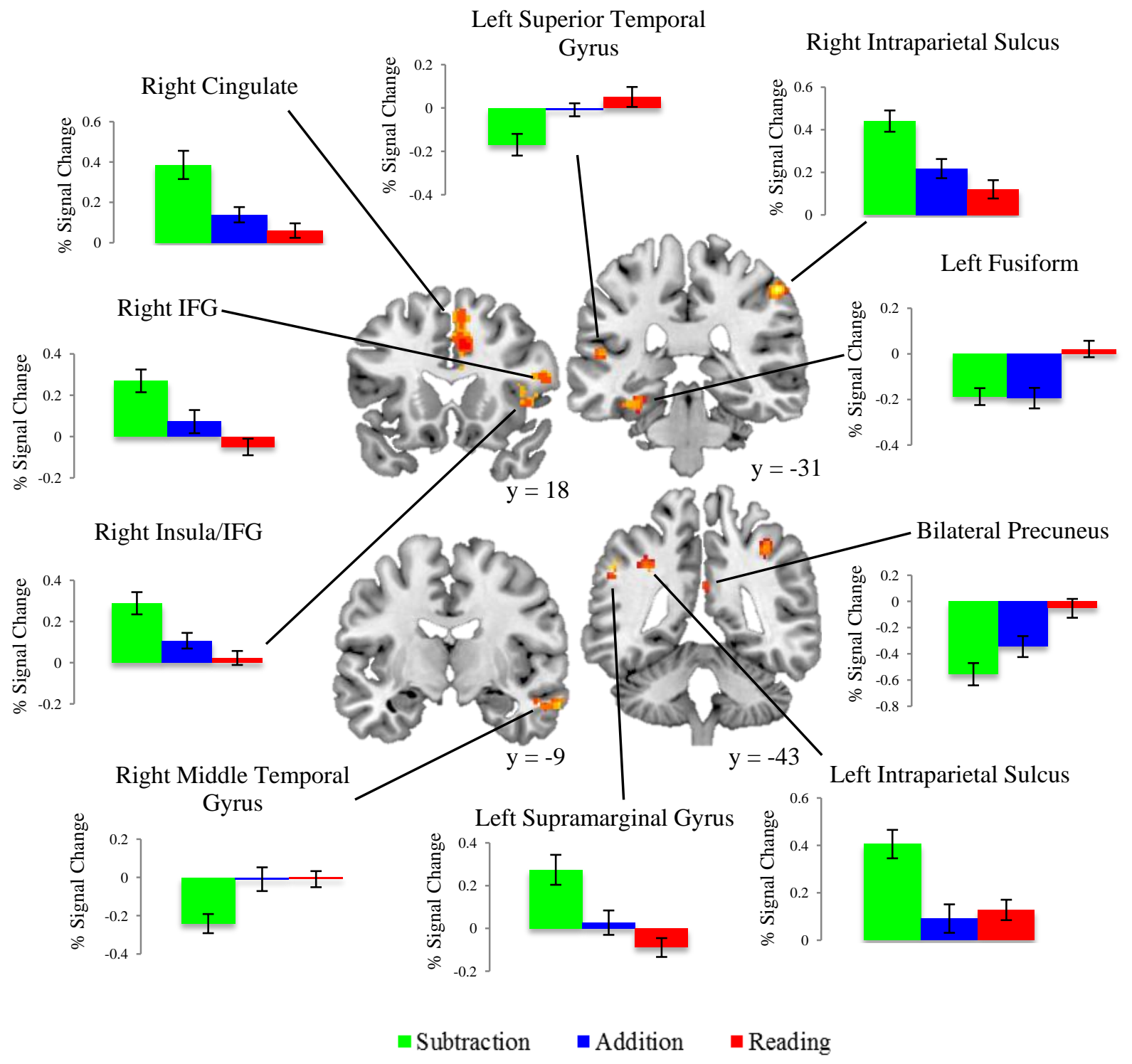

Figure 2 displays the Main Effect of Task from the factorial analysis (rendered onto coronal slices of a structural MRI scans at $y=18,-9,-31$, and -43). Percent signal change was extracted and is displayed as bar charts for each task (subtraction in green, addition in blue, reading in red); $p<0.005$ height threshold, $p<0.05$ cluster-level corrected extent threshold in the regions identified by the ANCOVA. 


\subsubsection{Main Effect of Age}

A main effect of Age was found in four clusters: right AG (PFm), superior temporal sulcus (STS; BA 21/22), and putamen, as well as left SMA (BA 6). All but the SMA showed increased activity with age, indicative of a developmental/experiential increase in right temporoparietal and sub-cortical circuitry for reading and arithmetic combined. However, left SMA showed a relative signal decrease with increasing age, indicative of a developmental decrease in frontal cortical circuitry. See Table 3 and Figure 3. 
Figure 3. Main Effect of Age
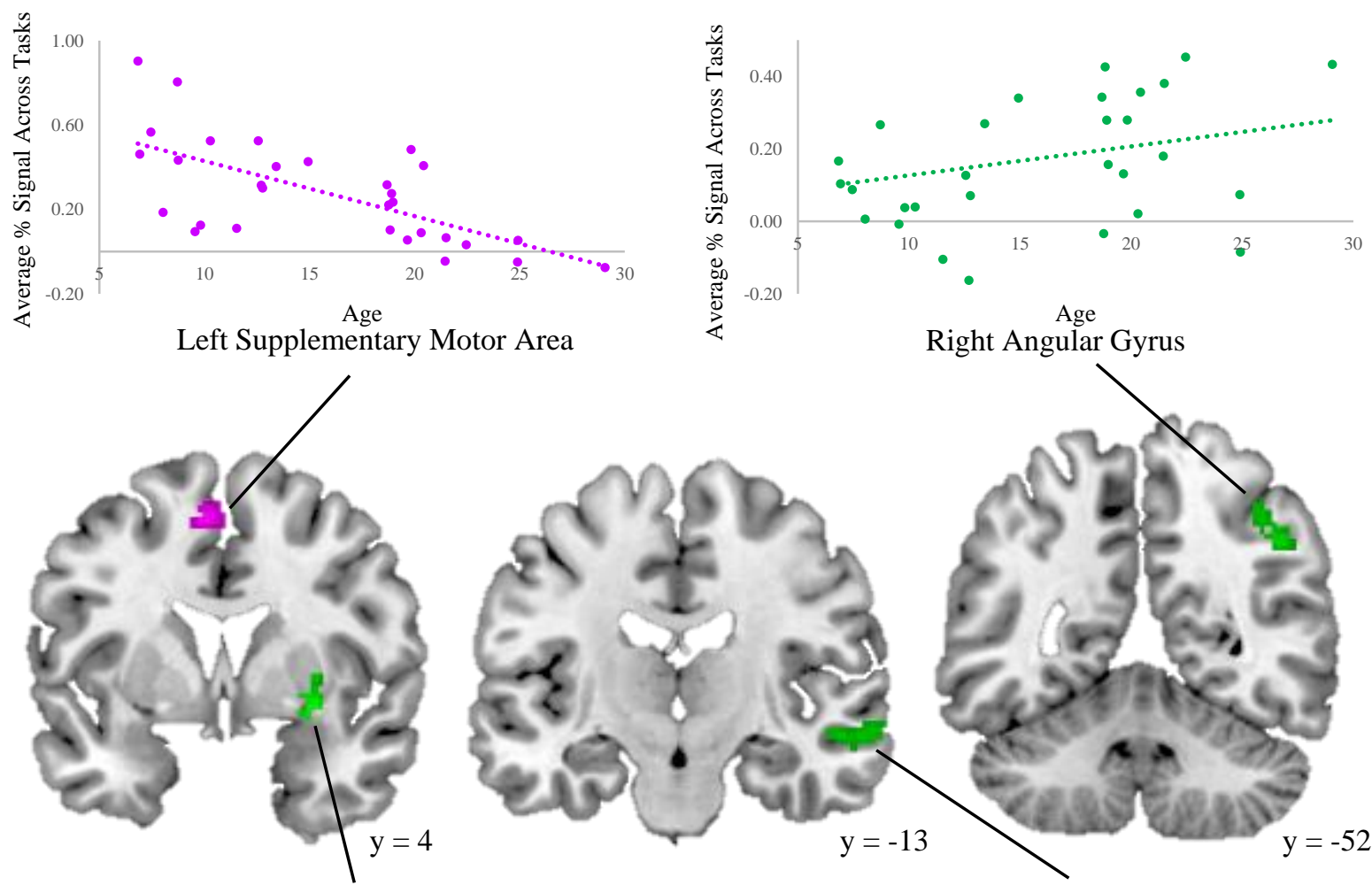

Right Angular Gyrus
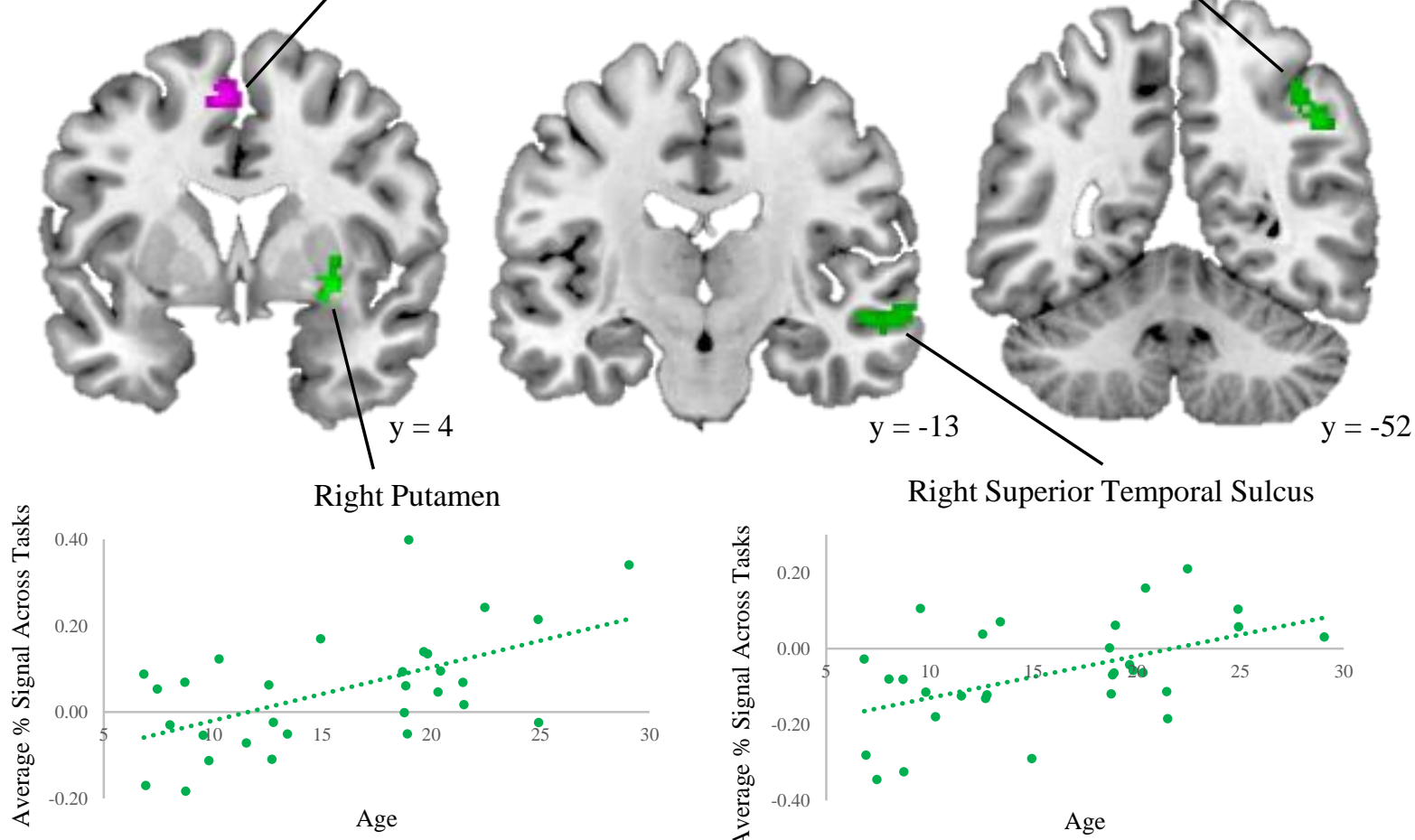

Right Superior Temporal Sulcus

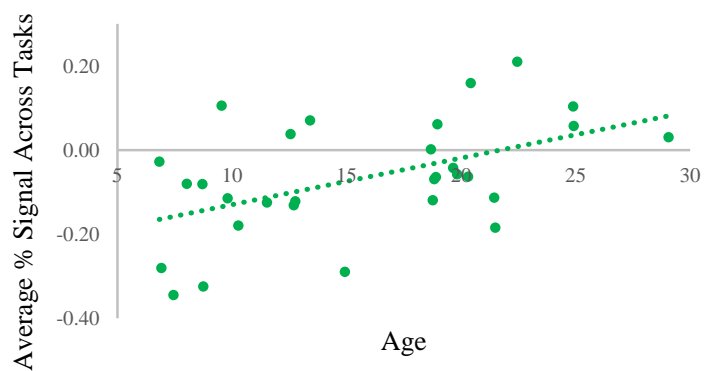

Figure 3 displays the Main Effect of Age from the factorial analysis; $p<0.005$ height threshold, $p<0.05$ cluster-level corrected extent threshold (rendered onto coronal slices of a structural MRI scan at $y=4,-13$, and -52). Positive effects are in green and negative effects are in violet. Scatter plots depict the extracted percent signal change plotted against age for all tasks combined. 


\subsubsection{Interaction: Task $x$ Age}

A Task x Age interaction was found in two clusters: right hippocampus and left MFG/IFG (BA 9). In both of these regions, increased activation was seen with increasing age for the addition task, while reading and subtraction instead were associated with decreased signal with increasing age. This provides evidence for a unique age-dependent pattern for the arithmetic operation of addition. See Table 3 and Figure 4.

\section{Figure 4. Task x Age Interaction}

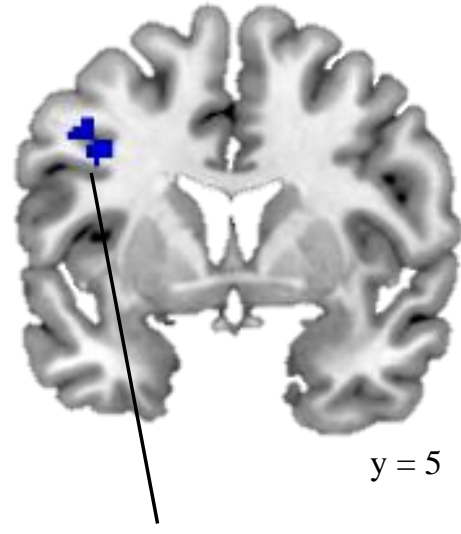

Left Middle/Inferior Frontal Gyrus

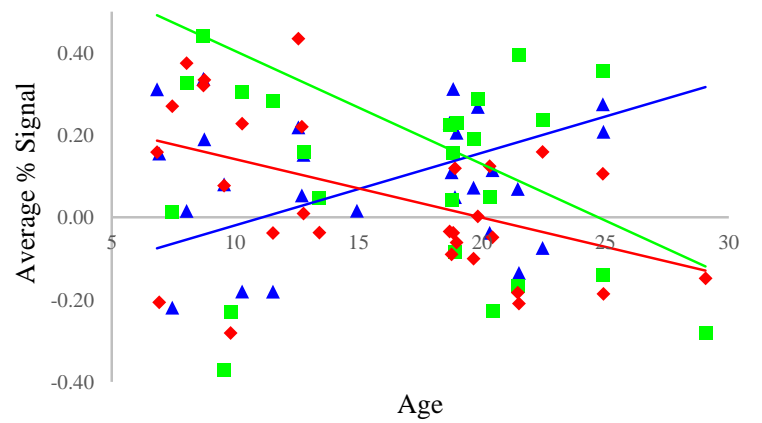

$\triangle$ Addition $\quad$ Subtraction $\bullet$ Reading

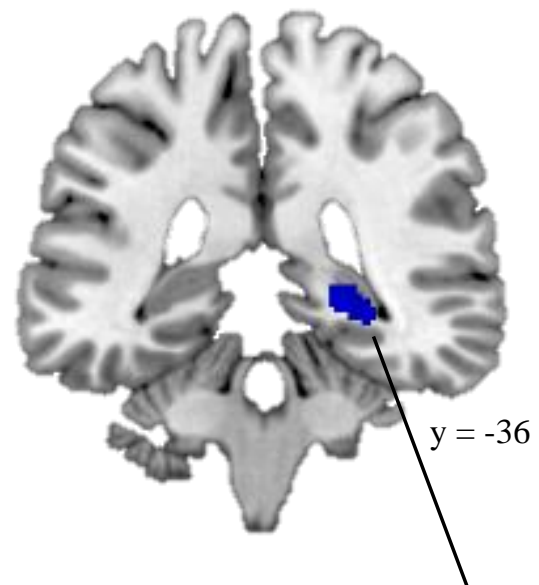

Right Hippocampus

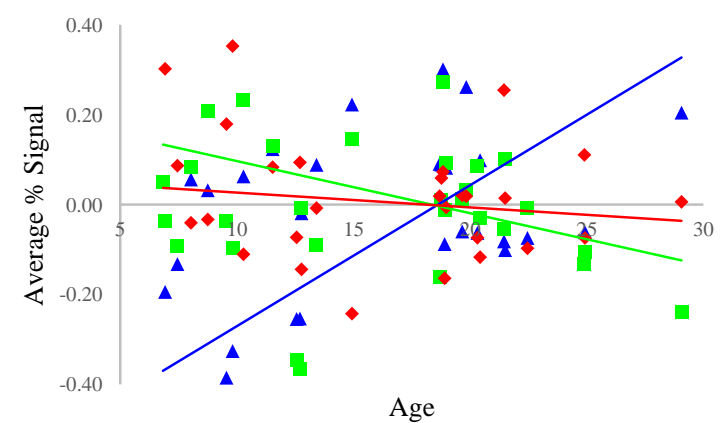

Age

Figure 4 displays the Task (subtraction task > fixation, addition task > fixation, reading task > fixation) $x$ Age interaction; $p<0.005$ height threshold, $p<0.05$ cluster-level corrected extent threshold rendered onto coronal slices of a structural MRI scan at $y=5$ and -36. Percent signal change vs. age for all subjects for subtraction (green regression line), addition (blue regression line), and reading (red regression line) tasks is displayed for each region. 
Table 3. Factorial Analysis: Task (Subtraction, Addition, Reading) x Age

\begin{tabular}{|c|c|c|c|c|c|}
\hline \multirow[t]{2}{*}{ Anatomical Region } & \multicolumn{3}{|c|}{$\begin{array}{c}\text { Peak MNI } \\
\text { Coordinates }\end{array}$} & \multirow[t]{2}{*}{ Z Score } & \multirow{2}{*}{$\begin{array}{c}\text { Volume } \\
\text { (voxels) } \\
\end{array}$} \\
\hline & $\mathbf{x}$ & $\mathbf{y}$ & $\mathbf{z}$ & & \\
\hline \multicolumn{6}{|l|}{ Main Effect of Task } \\
\hline Right intraparietal sulcus/supramarginal gyrus & 52 & -34 & 50 & 4.25 & 216 \\
\hline Right middle temporal gyrus & 58 & 0 & -18 & 3.75 & 121 \\
\hline Right insula/inferior frontal gyrus & 44 & 6 & 2 & 3.22 & 90 \\
\hline Right inferior frontal gyrus & 56 & 18 & 16 & 3.47 & 92 \\
\hline Right cingulate & 10 & 22 & 34 & 4.21 & 614 \\
\hline Left precuneus & -2 & -54 & 12 & 3.60 & 126 \\
\hline Left intraparietal sulcus & -36 & -48 & 44 & 4.03 & 90 \\
\hline Left supramarginal gyrus & -58 & -40 & 36 & 3.96 & 104 \\
\hline Left fusiform/parahippocampal gyrus & -34 & -34 & -18 & 3.61 & 114 \\
\hline Left superior temporal gyrus & -56 & -16 & 8 & 3.67 & 109 \\
\hline
\end{tabular}

Main Effect of Age

\begin{tabular}{lrrrrr}
\hline Right angular gyrus & 48 & -48 & 36 & 4.05 & 136 \\
\hline Right superior temporal sulcus & 62 & -22 & -6 & 3.50 & 172 \\
\hline Right putamen & 28 & 10 & 0 & 4.11 & 73 \\
\hline Left supplementary motor area & -6 & 6 & 54 & 4.01 & 72 \\
\hline
\end{tabular}

Interaction Task x Age

\begin{tabular}{lrrrrr}
\hline Right hippocampus & 22 & -36 & -2 & 3.31 & 78 \\
\hline Left middle/inferior frontal gyrus & -38 & 0 & 46 & 3.83 & 86 \\
\hline
\end{tabular}

\section{DISCUSSION}

In this study, we investigated the functional neuroanatomy of arithmetic, specifically for addition and subtraction of small numbers, as well as single-word reading, using closely matched tasks in children, adolescents, and adults. The purpose was to characterize the neural substrates that mediate these foundational classroom skills in typical subjects in the context of age/experience. We hypothesized subtraction to be subserved by bilateral frontal and parietal cortices, and addition and reading by left inferior parietal, middle/superior temporal, and inferior frontal cortices. Given the evidence for the disassociation between subtraction and addition, 
combined with the association between addition and reading from previous behavioral and neuroimaging studies, we expected to see different patterns of brain activity for subtraction and addition and similar left hemisphere activity for addition and reading. We anticipated taskrelated signal change to follow the posterior-to-anterior direction of the brain's developmental trajectory and at the same time be more pronounced with age due to maturational effects and experience: more activity in left inferior frontal cortex during reading and addition, and more activity in right parietal and frontal cortices during subtraction, with increasing age.

First, we found that in a group of 7- to 29-year-olds, tasks involving subtraction, addition, and reading resulted in the anticipated activity for each, consistent with the brain regions described in the published literature outlined in the introduction. Specifically, when compared with an active control condition, subtraction engaged bilateral fronto-parietal cortex and left cerebellum, addition engaged bilateral MFG and left IFG, and reading engaged left MTG/STG and IFG.

Next, the factorial analysis (using a common fixation baseline by which to compare all three tasks) revealed a main effect of Task in bilateral fronto-parietal cortex, which was more active during subtraction than during the other two tasks; in right middle temporal cortex and left STG, which were more active during both addition and reading relative to subtraction; and in left fusiform gyrus and precuneus, which were more active during reading than either of the arithmetic tasks. We found a main effect of Age in right AG, STS, and putamen, all of which were more active with increasing age when the tasks were considered jointly. A main effect of age was also found in left SMA, which was less active with increasing age. The interaction analysis of Task by Age identified the right hippocampus and left MFG/IFG, with activity here increasing with increasing age during addition and decreasing with increasing age during reading 
and subtraction. Together, these findings characterize the functional anatomy of arithmetic and reading over the period of childhood, adolescence, and young adulthood.

\subsection{Task-Specific Brain Activity for Arithmetic Operations and Reading Independent of Age}

Our experimental design used both addition and subtraction, allowing us to investigate the specific substrates for each arithmetic operation, and for the first time determine their relationship in the context of the functional anatomy of reading. Behavioral (Barrouillet et al., 2008; Campbell and Xue, 2001; Delazer et al., 2003a; Imbo and Vandierendonck, 2008) and neuroimaging studies (De Smedt et al., 2011; Rosenberg-Lee et al., 2011a) have revealed that arithmetic operations such as subtraction and addition of small numbers engage different mechanisms and hence unique brain substrates. However, a key study on the neurodevelopment of arithmetic processing combined addition and subtraction (Rivera et al., 2005), thereby losing the ability to examine task-specific regions of brain activity. Further, arithmetic and reading have not been simultaneously studied in children, with researchers instead relying on comparisons drawn from the published literature (Houdé et al., 2010). We expected to see similar activity patterns across the arithmetic operation of addition and reading, due to their shared cognitive mechanisms, and at the same time differential activity patterns for subtraction and reading, as supported by behavioral (De Smedt and Boets, 2010) and neuroimaging (Prado et al., 2011) studies.

We identified a main effect of Task in bilateral fronto-parietal cortex, which was engaged to a greater degree for subtraction relative to both addition and reading. This increased engagement could result from increased task difficulty for subtraction relative to addition and reading. Importantly, this result remained with or without reaction time entered as a covariate of no interest. This is in concordance with previous investigations that have identified the IPS in 
quantitative processing during arithmetic tasks in both adults (Dehaene and Cohen, 1997; Lee, 2000; Rosenberg-Lee et al., 2011a) and children (De Smedt et al., 2011; Rosenberg-Lee et al., 2011b), particularly for tasks including the operations of subtraction and division. Also, lesions to the right inferior parietal cortex manifest in quantitative arithmetic knowledge impairments (e.g., subtraction) but preserve rote arithmetic knowledge (e.g., addition; Dehaene and Cohen, 1997). Activity in IPS during calculation has been attributed to increased reliance on quantitative strategies (De Smedt et al., 2011; Delazer et al., 2003b; Rosenberg-Lee et al., 2011a). While the nature of our task does not allow us to exclude the possibility that subjects may have solved the subtraction problems by reverse addition (i.e., reading the problem from right to left) with simultaneous engagement of visual attention, our results are consistent with others using different paradigms (Rosenberg-Lee et al., 2011a) and different from those regions engaged during addition. Thus, we feel certain that the task elicited brain regions involved in subtraction.

We also identified a main effect of Task in the right middle temporal cortex and left STG, both of which were engaged to a greater degree for addition and reading, in both cases relative to subtraction. The engagement of left STG during word processing is consistent with prior studies using this specific task (Turkeltaub et al., 2003), other studies of reading (Jobard et al., 2003; Martin et al., 2015b), and a model of reading that points to temporo-parietal cortex as key in supporting skilled decoding (Price, 2012; Pugh et al., 2001). The left STG result also converges with the results of Prado and colleagues (2011), who identified via a localizer scan using a phonological processing task the MTG (near our STG location) and then found that within this region of interest, there was specificity for multiplication relative to subtraction. Multiplication, like addition, is thought to rely on verbal retrieval mechanisms also used for phonological processing. It is worth noting, however, that many (9 of 20) of the problems in our addition 
stimulus set include 1 as either the first or second operand. These ' +1 ' problems are viewed as special because of their likelihood of being solved with verbal counting and not quantitative strategies (Fayol and Thevenot, 2012), which could at least in part account for these results. As described above, our results link the underlying mechanisms to reading and phonological coding processes, which have been associated with specific aspects of arithmetic (De Smedt and Boets, 2010; Hecht et al., 2001; Simmons and Singleton, 2008). These studies are generally consistent with Dehaene's triple-code model of number processing (Dehaene, 1992; Dehaene and Cohen, 1995; Dehaene et al., 2003), suggesting that simple arithmetic fact retrieval in the addition of small numbers is supported by the verbal system housed in a network of brain regions, including temporal regions, and does not engage the complex processes required for quantitative procedures housed in the posterior parietal cortex (Dehaene et al., 2003). This languagedependent arithmetic coding is most likely the same as that responsible for the similarity of the response observed in the addition and reading tasks.

Lastly, we found a main effect of Task in the left fusiform gyrus and precuneus, which were preferentially engaged during the reading task relative to each arithmetic task. The result in the precuneus is not discussed further because despite being more active for reading than the arithmetic tasks, it was still below baseline. Our results reflect specific representations for words within the ventral visual processing stream in the left ventral occipito-temporal cortex (VOTC; Bruno et al., 2008; Cohen and Dehaene, 2004; Cohen et al., 2002; Dehaene et al., 2001; Kronbichler et al., 2004, 2007) and support a specialized role for a region within left VOTC (often deemed the 'Visual Word Form Area' or VWFA) in visual word identification (Cohen and Dehaene, 2004; Dehaene and Cohen, 2011; Glezer et al., 2009; McCandliss et al., 2003). Although subjects are actually explicitly solving mathematical problems in the math tasks, but 
not explicitly pronouncing words in the reading task, our results are consistent with the implicit processing of the word stimuli during this task. Although one might have expected VOTC to also be engaged during arithmetic processing (Dehaene and Cohen, 1995; Shum et al., 2013), either fMRI signal dropout (Grotheer et al., 2016) or more extensive experience with reading may account for increased activity for words relative to digits, and thus account for relatively more VOTC engagement during the reading task. Together, these results pinpoint substrates within the brain regions supporting arithmetic and reading that are unique to the specific demands of each task.

\subsection{Age-Related Brain Activity}

While the ultimate goal of this investigation was to establish the neural underpinnings of our reading and arithmetic tasks, because all of these skills depend on experience, it is obvious that any result is going to be highly dependent on the age of the participants. Independent developmental studies of arithmetic and reading have demonstrated an effect of age for each, as already described in the introduction. Our analysis for main effect of Age provides an opportunity to examine general age-dependent differences for all of the tasks used here, though notably does not parse out experience-dependent from age-dependent change. We expected that adults would engage bilateral parietal and/or frontal cortices to a greater extent than children based on prior studies of arithmetic (Kawashima et al., 2004; Kucian et al., 2008; Rivera et al., 2005) and reading (Schlaggar and McCandliss, 2007; Turkeltaub et al., 2003) and based on the posterior-to-anterior direction of brain maturation (Huttenlocher, 1990).

Indeed, we found a main effect of Age in posterior temporo-parietal cortex, specifically right AG and STS, with individuals exhibiting greater activity in both regions with increasing age across all tasks (subtraction, addition, and reading). Within anterior brain regions, we found 
a positive effect of age in the putamen and a negative effect of age in SMA activity. These regions all undergo dynamic changes over the course of development, as demonstrated by previous developmental studies of neuroanatomy over this age range. Notably, this work has identified dramatic decreases in gray matter density (indicative of maturation through pruning) in dorsal aspects of frontal cortex, and subtle increases in bilateral posterior temporal and inferior parietal lobes from ages 7-30 (Sowell et al., 2003; Toga et al., 2006).

The posterior results in right AG and STS are consistent with increased involvement with age for regions involved in symbolic mapping and language processing and previously studied in isolation, as will be discussed next. Studies using exact arithmetic tasks (addition in Kucian et al., 2008; addition and subtraction in Kawashima et al., 2004, and Rivera et al., 2005) in schoolaged children and adults (N.B. Rivera et al. was cross-sectional) have found age-specific increases in bilateral parietal (Kawashima et al., 2004; Kucian et al., 2008; Rivera et al., 2005) and frontal (Kawashima et al., 2004; Kucian et al., 2008) cortices. These studies support the theory that there is greater engagement of posterior parietal cortices with age as calculations become more automated (Menon, 2010; Rivera et al., 2005). Similarly, developmental studies of reading have shown increases in parietal cortex with increasing age. For example, Bitan and colleagues show increased posterior parietal cortex engagement from ages 9 to 15 during a phonological processing task (Bitan et al., 2007).

In anterior regions we found an increase in the use of the right putamen with age, which could reflect an increased involvement across development of automatized procedural memory circuitry (Kreitzer and Malenka, 2008). Our finding of decreased reliance on SMA with age is consistent with studies reporting the same pattern in SMA activity in a cross-sectional sample during arithmetic processing (Rivera et al., 2005) and meta-analyses of reading in children and 
adults (Martin et al., 2015b), and could reflect increased neural efficiency in this region.

Together, our results across all tasks support the theory of increased use of parietal cortex with age/experience and further suggest a cortical to subcortical shift within anterior brain regions with age.

\subsection{Task-Specific and Age-Dependent Brain Activity for Reading and Arithmetic}

To date, the only evidence for age-dependent changes (children versus young adults) that are specific to reading and arithmetic come from a meta-analysis by Houdé and colleagues (Houdé et al., 2010), who concluded, based on the analysis of published work, that while reading induces similar activity in children and adults, numerical processing tasks activate the frontal regions of children more than adults. The interaction analysis allowed us to directly identify Task by Age interactions, and we found these to occur in the right hippocampus as well as the left MFG/IFG. Both areas were relatively less active in children than adults during addition, and at the same more active in children relative to adults during both subtraction and reading. As such, our data are partially consistent with the conclusions from the meta-analysis, with subtraction activating the frontal regions in MFG/IFG of children relatively more than adults. Houdé and colleagues concluded that reading-induced brain activity is similar in children and adults, and our results concur, other than the two foci reported here: more activity in children during reading in the right hippocampus and the left MFG/IFG. The latter finding countered our own expectations, as we had predicted more activity in left inferior frontal cortex during reading and addition in adults, not children.

The strong increase of activity for addition with increasing age in the right hippocampus was striking. At the same time, activity here was less in adults than children for addition and subtraction. Previous work has shown the hippocampus to be involved in arithmetic tasks (De 
Smedt et al., 2011; Qin, 2014; Rivera et al., 2005). Notably, the hippocampus is more active during addition relative to subtraction arithmetic problems (De Smedt et al., 2011; Delazer et al., 2003b), and increases in hippocampal activity have been demonstrated in longitudinal studies in childhood during addition, though less activity was found in adults in the same study (Qin et al., 2014). Decreases in hippocampal activity with increasing age were observed in a study that combined addition and subtraction (Rivera et al., 2005). Our results suggest that addition and subtraction follow opposite developmental trajectories in the hippocampus, and that reading is similar to subtraction in this regard. The hippocampus has been posited to play a role in declarative memory, which may play a compensatory role in childhood disorders, such as specific language impairment, dyslexia, and autism spectrum disorder (Ullman and Pullman, 2015). These results suggest that addition may continue to require increased retrieval resources into adulthood, while subtraction and reading may not, thereby underscoring the fact that these age- and task-dependent relationships need to be considered in the context of learning disabilities.

Previous work has demonstrated that left MFG activity (more anterior to that reported here), within dorsolateral prefrontal cortex, increases with age during addition problem solving from second to third grade (Rosenberg-Lee et al., 2011b). This together with the finding presented here strongly supports an age-dependent effect specific to addition. Whilst one might have anticipated the same age-dependent increase for reading, as already mentioned, the decrease of activity in left MFG/IFG with increasing age came as a surprise, as previous work from our laboratory using this same implicit reading task in a different group of participants age 6 to 22 years of age, revealed increasing left IFG activity with increasing age, as well as with stronger reading ability (Turkeltaub et al., 2003). 


\subsection{Conclusion}

Our results contribute to the understanding of the neural bases of arithmetic and reading in childhood, adolescence, and into early adulthood. We identify the unique brain systems that support these educationally important and culturally driven skills relative to one another and in the context of age. We provide further support for divergence (reading and subtraction), as well as some degree of convergence (reading and addition), between these networks, independent of age/experience. Therefore, our results add to studies conducted in adults arguing for a brainbased connection between language and arithmetic (Dehaene and Cohen, 1995; Dehaene et al., 2003; Prado et al., 2011) and extend this to children. At the same time we found interactions between task and age, highlighting the need for future studies to take a longitudinal approach.

\section{ACKNOWLEDGEMENTS}

This work was supported by the National Institute of Child Health and Human Development (P50HD40095 and R01HD056107) and the National Science Foundation (SBE0541953 Science of Learning Center). We are grateful to the staff at the Center for Functional and Molecular Imaging, and for the support of the Intellectual and Development Disorders Research Center (IDDRC5P30HD040677-13). Thanks to the following for aiding in the acquisition of behavioral and MRI data: Erin Ingala, Emma Cole, Iain DeWitt, Alison Merikangas, Jenni Rosenberg, and Ashley Wall-Piche. We also thank Theodore Turesky, Olumide Olulade, Diana Alkire, Jamie Neiman, Miriam Rosenberg-Lee, and Neha Vellanki for their help, and each of our participants for their time. We are grateful to three anonymous reviewers for their constructive feedback. 


\section{REFERENCES}

Anderson, S.W., Damasio, A.R., and Damasio, H. (1990). Troubled letters but not numbers. Domain specific cognitive impairments following focal damage in frontal cortex. Brain J. Neurol. 113 ( Pt 3), 749-766.

Arsalidou, M., and Taylor, M.J. (2011). Is $2+2=4$ ? Meta-analyses of brain areas needed for numbers and calculations. NeuroImage 54, 2382-2393.

Barnes, M.A., Raghubar, K.P., English, L., Williams, J.M., Taylor, H., and Landry, S. (2014). Longitudinal mediators of achievement in mathematics and reading in typical and atypical development. J. Exp. Child Psychol. 119, 1-16.

Barrouillet, P., Mignon, M., and Thevenot, C. (2008). Strategies in subtraction problem solving in children. J. Exp. Child Psychol. 99, 233-251.

Bitan, T., Cheon, J., Lu, D., Burman, D.D., Gitelman, D.R., Mesulam, M.-M., and Booth, J.R. (2007). Developmental changes in activation and effective connectivity in phonological processing. NeuroImage 38, 564-575.

Brett, M., Anton, J.-L., Valabregue, R., and Poline, J.-B. (2002). Region of interest analysis using an SPM toolbox. (Sendai, Japan), p.

Bruno, J.L., Zumberge, A., Manis, F.R., Lu, Z.-L., and Goldman, J.G. (2008). Sensitivity to orthographic familiarity in the occipito-temporal region. NeuroImage 39, 1988-2001.

Burgund, E.D., Kang, H.C., Kelly, J.E., Buckner, R.L., Snyder, A.Z., Petersen, S.E., and Schlaggar, B.L. (2002). The feasibility of a common stereotactic space for children and adults in fMRI studies of development. NeuroImage 17, 184-200.

Campbell, J.I., and Xue, Q. (2001). Cognitive arithmetic across cultures. J. Exp. Psychol. Gen. $130,299-315$.

Caspers, S., Geyer, S., Schleicher, A., Mohlberg, H., Amunts, K., and Zilles, K. (2006). The human inferior parietal cortex: Cytoarchitectonic parcellation and interindividual variability. NeuroImage 33, 430-448.

Choi, H.-J., Zilles, K., Mohlberg, H., Schleicher, A., Fink, G.R., Armstrong, E., and Amunts, K. (2006). Cytoarchitectonic identification and probabilistic mapping of two distinct areas within the anterior ventral bank of the human intraparietal sulcus. J. Comp. Neurol. 495, 53-69.

Church, J.A., Coalson, R.S., Lugar, H.M., Petersen, S.E., and Schlaggar, B.L. (2008). A developmental fMRI study of reading and repetition reveals changes in phonological and visual mechanisms over age. Cereb. Cortex N. Y. N 1991 18, 2054-2065.

Church, J.A., Petersen, S.E., and Schlaggar, B.L. (2010). The "Task B problem" and other considerations in developmental functional neuroimaging. Hum. Brain Mapp. 31, 852-862. 
Cohen, L., and Dehaene, S. (2004). Specialization within the ventral stream: the case for the visual word form area. NeuroImage 22, 466-476.

Cohen, L., Dehaene, S., Chochon, F., Lehéricy, S., and Naccache, L. (2000). Language and calculation within the parietal lobe: a combined cognitive, anatomical and fMRI study. Neuropsychologia 38, 1426-1440.

Cohen, L., Lehéricy, S., Chochon, F., Lemer, C., Rivaud, S., and Dehaene, S. (2002). Languagespecific tuning of visual cortex? Functional properties of the Visual Word Form Area. Brain J. Neurol. 125, 1054-1069.

De Smedt, B., and Boets, B. (2010). Phonological processing and arithmetic fact retrieval: evidence from developmental dyslexia. Neuropsychologia 48, 3973-3981.

De Smedt, B., Taylor, J., Archibald, L., and Ansari, D. (2010). How is phonological processing related to individual differences in children's arithmetic skills? Dev. Sci. 13, 508-520.

De Smedt, B., Holloway, I.D., and Ansari, D. (2011). Effects of problem size and arithmetic operation on brain activation during calculation in children with varying levels of arithmetical fluency. NeuroImage 57, 771-781.

Dehaene, S. (1992). Varieties of numerical abilities. Cognition 44, 1-42.

Dehaene, S., and Cohen, L. (1995). Towards an anatomical and functional model of number processing. Math. Cogn. 1, 83-120.

Dehaene, S., and Cohen, L. (1997). Cerebral pathways for calculation: double dissociation between rote verbal and quantitative knowledge of arithmetic. Cortex J. Devoted Study Nerv. Syst. Behav. 33, 219-250.

Dehaene, S., and Cohen, L. (2007). Cultural recycling of cortical maps. Neuron 56, 384-398.

Dehaene, S., and Cohen, L. (2011). The unique role of the visual word form area in reading. Trends Cogn. Sci. 15, 254-262.

Dehaene, S., Naccache, L., Cohen, L., Bihan, D.L., Mangin, J.F., Poline, J.B., and Rivière, D. (2001). Cerebral mechanisms of word masking and unconscious repetition priming. Nat. Neurosci. 4, 752-758.

Dehaene, S., Piazza, M., Pinel, P., and Cohen, L. (2003). Three parietal circuits for number processing. Cogn. Neuropsychol. 20, 487-506.

Delazer, M., Girelli, L., Granà, A., and Domahs, F. (2003a). Number processing and calculation-normative data from healthy adults. Clin. Neuropsychol. 17, 331-350.

Delazer, M., Domahs, F., Bartha, L., Brenneis, C., Lochy, A., Trieb, T., and Benke, T. (2003b). Learning complex arithmetic--an fMRI study. Brain Res. Cogn. Brain Res. 18, 76-88. 
Dunst, B., Benedek, M., Jauk, E., Bergner, S., Koschutnig, K., Sommer, M., Ischebeck, A., Spinath, B., Arendasy, M., Bühner, M., et al. (2014). Neural efficiency as a function of task demands. Intelligence 42, 22-30.

Eickhoff, S.B., Stephan, K.E., Mohlberg, H., Grefkes, C., Fink, G.R., Amunts, K., and Zilles, K. (2005). A new SPM toolbox for combining probabilistic cytoarchitectonic maps and functional imaging data. NeuroImage 25, 1325-1335.

Eickhoff, S.B., Paus, T., Caspers, S., Grosbras, M.-H., Evans, A.C., Zilles, K., and Amunts, K. (2007). Assignment of functional activations to probabilistic cytoarchitectonic areas revisited. NeuroImage 36, 511-521.

Evans, T.M., Lynn Flowers, D., Napoliello, E.M., Olulade, O.A., and Eden, G.F. (2014). The functional anatomy of single-digit arithmetic in children with developmental dyslexia. NeuroImage.

Fayol, M., and Thevenot, C. (2012). The use of procedural knowledge in simple addition and subtraction problems. Cognition 123, 392-403.

Giedd, J.N., Blumenthal, J., Jeffries, N.O., Castellanos, F.X., Liu, H., Zijdenbos, A., Paus, T., Evans, A.C., and Rapoport, J.L. (1999). Brain development during childhood and adolescence: a longitudinal MRI study. Nat. Neurosci. 2, 861-863.

Glezer, L.S., Jiang, X., and Riesenhuber, M. (2009). Evidence for highly selective neuronal tuning to whole words in the "visual word form area." Neuron 62, 199-204.

Grabner, R.H., Ansari, D., Reishofer, G., Stern, E., Ebner, F., and Neuper, C. (2007). Individual differences in mathematical competence predict parietal brain activation during mental calculation. NeuroImage 38, 346-356.

Grabner, R.H., Ansari, D., Koschutnig, K., Reishofer, G., Ebner, F., and Neuper, C. (2009). To retrieve or to calculate? Left angular gyrus mediates the retrieval of arithmetic facts during problem solving. Neuropsychologia 47, 604-608.

Grotheer, M., Herrmann, K.-H., and Kovács, G. (2016). Neuroimaging Evidence of a Bilateral Representation for Visually Presented Numbers. J. Neurosci. 36, 88-97.

van Harskamp, N.J., and Cipolotti, L. (2001). Selective impairments for addition, subtraction and multiplication. implications for the organisation of arithmetical facts. Cortex J. Devoted Study Nerv. Syst. Behav. 37, 363-388.

van Harskamp, N.J., Rudge, P., and Cipolotti, L. (2002). Are multiplication facts implemented by the left supramarginal and angular gyri? Neuropsychologia 40, 1786-1793.

Hecht, S.A., Torgesen, J.K., Wagner, R.K., and Rashotte, C.A. (2001). The relations between phonological processing abilities and emerging individual differences in mathematical computation skills: a longitudinal study from second to fifth grades. J. Exp. Child Psychol. 79, $192-227$. 
Houdé, O., Rossi, S., Lubin, A., and Joliot, M. (2010). Mapping numerical processing, reading, and executive functions in the developing brain: an fMRI meta-analysis of 52 studies including 842 children. Dev. Sci. 13, 876-885.

Huttenlocher, P.R. (1990). Morphometric study of human cerebral cortex development. Neuropsychologia 28, 517-527.

Imbo, I., and Vandierendonck, A. (2008). Effects of problem size, operation, and workingmemory span on simple-arithmetic strategies: differences between children and adults? Psychol. Res. 72, 331-346.

Jobard, G., Crivello, F., and Tzourio-Mazoyer, N. (2003). Evaluation of the dual route theory of reading: a metanalysis of 35 neuroimaging studies. NeuroImage 20, 693-712.

Kang, H.C., Burgund, E.D., Lugar, H.M., Petersen, S.E., and Schlaggar, B.L. (2003). Comparison of functional activation foci in children and adults using a common stereotactic space. NeuroImage 19, 16-28.

Kaufmann, L., Wood, G., Rubinsten, O., and Henik, A. (2011). Meta-analyses of developmental fMRI studies investigating typical and atypical trajectories of number processing and calculation. Dev. Neuropsychol. 36, 763-787.

Kawashima, R., Taira, M., Okita, K., Inoue, K., Tajima, N., Yoshida, H., Sasaki, T., Sugiura, M., Watanabe, J., and Fukuda, H. (2004). A functional MRI study of simple arithmetic--a comparison between children and adults. Brain Res. Cogn. Brain Res. 18, 227-233.

Krafnick, A.J., Tan, L.-H., Flowers, D.L., Luetje, M.M., Napoliello, E.M., Siok, W.-T., Perfetti, C., and Eden, G.F. (2016). Chinese Character and English Word processing in children's ventral occipitotemporal cortex: fMRI evidence for script invariance. NeuroImage 133, 302-312.

Kreitzer, A.C., and Malenka, R.C. (2008). Striatal plasticity and basal ganglia circuit function. Neuron 60, 543-554.

Kronbichler, M., Hutzler, F., Wimmer, H., Mair, A., Staffen, W., and Ladurner, G. (2004). The visual word form area and the frequency with which words are encountered: evidence from a parametric fMRI study. NeuroImage 21, 946-953.

Kronbichler, M., Bergmann, J., Hutzler, F., Staffen, W., Mair, A., Ladurner, G., and Wimmer, H. (2007). Taxi vs. taksi: on orthographic word recognition in the left ventral occipitotemporal cortex. J. Cogn. Neurosci. 19, 1584-1594.

Kucian, K., von Aster, M., Loenneker, T., Dietrich, T., and Martin, E. (2008). Development of neural networks for exact and approximate calculation: a FMRI study. Dev. Neuropsychol. 33, $447-473$.

Lee, K.M. (2000). Cortical areas differentially involved in multiplication and subtraction: a functional magnetic resonance imaging study and correlation with a case of selective acalculia. Ann. Neurol. 48, 657-661. 
Lee, K.-M., and Kang, S.-Y. (2002). Arithmetic operation and working memory: differential suppression in dual tasks. Cognition 83, B63-68.

Lewandowska, M., Piatkowska-Janko, E., Bogorodzki, P., Wolak, T., and Szelag, E. (2010). Changes in fMRI BOLD response to increasing and decreasing task difficulty during auditory perception of temporal order. Neurobiol. Learn. Mem. 94, 382-391.

Lu, L., Leonard, C., Thompson, P., Kan, E., Jolley, J., Welcome, S., Toga, A., and Sowell, E. (2007). Normal developmental changes in inferior frontal gray matter are associated with improvement in phonological processing: a longitudinal MRI analysis. Cereb. Cortex N. Y. N 1991 17, 1092-1099.

Martin, A., Schurz, M., Kronbichler, M., and Richlan, F. (2015a). Reading in the brain of children and adults: a meta-analysis of 40 functional magnetic resonance imaging studies. Hum. Brain Mapp. 36, 1963-1981.

Martin, A., Schurz, M., Kronbichler, M., and Richlan, F. (2015b). Reading in the brain of children and adults: A meta-analysis of 40 functional magnetic resonance imaging studies. Hum. Brain Mapp. 36, 1963-1981.

McCandliss, B.D., Cohen, L., and Dehaene, S. (2003). The visual word form area: expertise for reading in the fusiform gyrus. Trends Cogn. Sci. 7, 293-299.

Melby-Lervåg, M., Lyster, S.-A.H., and Hulme, C. (2012). Phonological skills and their role in learning to read: a meta-analytic review. Psychol. Bull. 138, 322-352.

Menon, V. (2010). Developmental cognitive neuroscience of arithmetic: implications for learning and education. ZDM Int. J. Math. Educ. 42, 515-525.

Mihulowicz, U., Willmes, K., Karnath, H.-O., and Klein, E. (2014). Single-digit arithmetic processing-anatomical evidence from statistical voxel-based lesion analysis. Front. Hum. Neurosci. 8, 286.

Nichols, T., and Hayasaka, S. (2003). Controlling the familywise error rate in functional neuroimaging: a comparative review. Stat. Methods Med. Res. 12, 419-446.

Nieder, A., and Dehaene, S. (2009). Representation of number in the brain. Annu. Rev. Neurosci. $32,185-208$.

Odegard, T.N., Farris, E.A., Ring, J., McColl, R., and Black, J. (2009). Brain connectivity in non-reading impaired children and children diagnosed with developmental dyslexia.

Neuropsychologia 47, 1972-7.

Olulade, O.A., Flowers, D.L., Napoliello, E.M., and Eden, G.F. (2013a). Developmental differences for word processing in the ventral stream. Brain Lang. 125, 134-145.

Olulade, O.A., Flowers, D.L., Napoliello, E.M., and Eden, G.F. (2013b). Developmental differences for word processing in the ventral stream. Brain Lang. 125, 134-145. 
Olulade, O.A., Napoliello, E.M., and Eden, G.F. (2013c). Abnormal visual motion processing is not a cause of dyslexia. Neuron 79, 180-190.

Olulade, O.A., Flowers, D.L., Napoliello, E.M., and Eden, G.F. (2015). Dyslexic children lack word selectivity gradients in occipito-temporal and inferior frontal cortex. NeuroImage Clin. 7, $742-754$.

Paulesu, E., Démonet, J.F., Fazio, F., McCrory, E., Chanoine, V., Brunswick, N., Cappa, S.F., Cossu, G., Habib, M., Frith, C.D., et al. (2001). Dyslexia: cultural diversity and biological unity. Science 291, 2165-2167.

Prado, J., Mutreja, R., Zhang, H., Mehta, R., Desroches, A.S., Minas, J.E., and Booth, J.R. (2011). Distinct representations of subtraction and multiplication in the neural systems for numerosity and language. Hum. Brain Mapp. 32, 1932-1947.

Price, C.J. (2012). A review and synthesis of the first 20 years of PET and fMRI studies of heard speech, spoken language and reading. NeuroImage 62, 816-847.

Price, C.J., Wise, R.J., and Frackowiak, R.S. (1996). Demonstrating the implicit processing of visually presented words and pseudowords. Cereb. Cortex N. Y. N 1991 6, 62-70.

Pugh, K.R., Mencl, W.E., Jenner, A.R., Katz, L., Frost, S.J., Lee, J.R., Shaywitz, S.E., and Shaywitz, B.A. (2001). Neurobiological studies of reading and reading disability. J. Commun. Disord. 34, 479-492.

Qin, S. (2014). Hippocampal-neocortical functional reorganization underlies children's cognitive development. Nat. Neurosci. in press.

Qin, S., Cho, S., Chen, T., Rosenberg-Lee, M., Geary, D.C., and Menon, V. (2014).

Hippocampal-neocortical functional reorganization underlies children's cognitive development. Nat. Neurosci. 17, 1263-1269.

Rauschecker, A.M., Deutsch, G.K., Ben-Shachar, M., Schwartzman, A., Perry, L.M., and Dougherty, R.F. (2009). Reading impairment in a patient with missing arcuate fasciculus. Neuropsychologia 47, 180-194.

Rivera, S.M., Reiss, A.L., Eckert, M.A., and Menon, V. (2005). Developmental changes in mental arithmetic: evidence for increased functional specialization in the left inferior parietal cortex. Cereb. Cortex N. Y. N 1991 15, 1779-1790.

Rosenberg-Lee, M., Chang, T.T., Young, C.B., Wu, S., and Menon, V. (2011a). Functional dissociations between four basic arithmetic operations in the human posterior parietal cortex: a cytoarchitectonic mapping study. Neuropsychologia 49, 2592-2608.

Rosenberg-Lee, M., Barth, M., and Menon, V. (2011b). What difference does a year of schooling make? Maturation of brain response and connectivity between 2nd and 3rd grades during arithmetic problem solving. NeuroImage 57, 796-808. 
Schlaggar, B.L., and McCandliss, B.D. (2007). Development of neural systems for reading. Annu. Rev. Neurosci. 30, 475-503.

Schlaggar, B.L., Brown, T.T., Lugar, H.M., Visscher, K.M., Miezin, F.M., and Petersen, S.E. (2002). Functional neuroanatomical differences between adults and school-age children in the processing of single words. Science 296, 1476-1479.

Shaw, P., Greenstein, D., Lerch, J., Clasen, L., Lenroot, R., Gogtay, N., Evans, A., Rapoport, J., and Giedd, J. (2006). Intellectual ability and cortical development in children and adolescents. Nature 440, 676-679.

Shum, J., Hermes, D., Foster, B.L., Dastjerdi, M., Rangarajan, V., Winawer, J., Miller, K.J., and Parvizi, J. (2013). A Brain Area for Visual Numerals. J. Neurosci. 33, 6709-6715.

Simmons, F.R., and Singleton, C. (2008). Do weak phonological representations impact on arithmetic development? A review of research into arithmetic and dyslexia. Dyslexia Chichester Engl. 14, 77-94.

Sowell, E.R., Peterson, B.S., Thompson, P.M., Welcome, S.E., Henkenius, A.L., and Toga, A.W. (2003). Mapping cortical change across the human life span. Nat. Neurosci. 6, 309-315.

Thiebaut de Schotten, M., Cohen, L., Amemiya, E., Braga, L.W., and Dehaene, S. (2012). Learning to Read Improves the Structure of the Arcuate Fasciculus. Cereb. Cortex N. Y. N 1991.

Toga, A.W., Thompson, P.M., and Sowell, E.R. (2006). Mapping brain maturation. Trends Neurosci. 29, 148-159.

Turkeltaub, P.E., Gareau, L., Flowers, D.L., Zeffiro, T.A., and Eden, G.F. (2003). Development of neural mechanisms for reading. Nat. Neurosci. 6, 767-773.

Turkeltaub, P.E., Flowers, D.L., Verbalis, A., Miranda, M., Gareau, L., and Eden, G.F. (2004). The neural basis of hyperlexic reading: an FMRI case study. Neuron 41, 11-25.

Ullman, M.T., and Pullman, M.Y. (2015). A compensatory role for declarative memory in neurodevelopmental disorders. Neurosci. Biobehav. Rev. 51, 205-222.

Van Beek, L., Ghesquière, P., Lagae, L., and De Smedt, B. (2014). Left fronto-parietal white matter correlates with individual differences in children's ability to solve additions and multiplications: a tractography study. NeuroImage 90, 117-127.

Vandermosten, M., Boets, B., Wouters, J., and Ghesquière, P. (2012). A qualitative and quantitative review of diffusion tensor imaging studies in reading and dyslexia. Neurosci. Biobehav. Rev. 36, 1532-1552.

Vogan, V.M., Morgan, B.R., Powell, T.L., Smith, M.L., and Taylor, M.J. (2016). The neurodevelopmental differences of increasing verbal working memory demand in children and adults. Dev. Cogn. Neurosci. 17, 19-27. 
Vogel, A.C., Church, J.A., Power, J.D., Miezin, F.M., Petersen, S.E., and Schlaggar, B.L. (2013). Functional network architecture of reading-related regions across development. Brain Lang. 125, 231-243.

Wechsler, D. (1999). Wechsler abbreviated scale of intelligence.

Wilke, M., Schmithorst, V.J., and Holland, S.K. (2002). Assessment of spatial normalization of whole-brain magnetic resonance images in children. Hum. Brain Mapp. 17, 48-60.

Wilke, M., Schmithorst, V. j., and Holland, S. k. (2003). Normative pediatric brain data for spatial normalization and segmentation differs from standard adult data. Magn. Reson. Med. 50, 749-757.

Yeatman, J.D., Dougherty, R.F., Rykhlevskaia, E., Sherbondy, A.J., Deutsch, G.K., Wandell, B.A., and Ben-Shachar, M. (2011). Anatomical properties of the arcuate fasciculus predict phonological and reading skills in children. J. Cogn. Neurosci. 23, 3304-3317. 